\title{
Análisis crítico del impuesto nacional al consumo de bolsas plásticas*
}

\section{Critical analysis of the National Consumption Tax on Plastic Bags}

\author{
YHONY ALBERTO LEE YARA ${ }^{1}$
}

\section{Sumario}

Introducción. I. Hecho generador. II. Sujeto pasivo económico. III. Sujeto pasivo jurídico. IV. Base gravable. V. La tarifa. VI. El elemento espacial. VII. Otras consideraciones. 1. Contabilización y su relación con el impuesto sobre la Renta. 2. La factura o documento equivalente. 3. Monotributo. 4. Declaración. 5. Actualización del RUT. 6. El cambio comercial. 7. Régimen sancionatorio. VIII. Conclusiones. Bibliografía.

\section{Resumen}

La Ley 1819 de 2016 en sus artículos 207 y 208 reguló en su integridad el Impuesto Nacional al Consumo de Bolsas Plásticas (en adelante INCBP). La propuesta de este nuevo tributo no fue invención del Gobierno Nacional, ya que no se consideró en el pro- yecto inicialmente presentado, sino que tuvo como creadores originales al partido Alianza Verde, y específicamente el senador Antonio Navarro Wolf ${ }^{2}$, y al partido Centro Democrático; este último lo concibió como una tasa para la promoción del medioambiente ${ }^{3}$. Finalmente, se incluyó en la ponencia de primer debate como un impuesto, evitando así una habilidosidad que eventualmente pudiese convertirse en una inconstitucionalidad de este impuesto (Lee Yara, www.gerencie. com, 2017).

El INCBP parecería un impuesto superfluo, ya que gran parte de las discusiones son consideradas estériles, toda vez que los sujetos pasivos lo reducen a una discusión de \$20. No obstante, como lo demostraremos a lo largo de este escrito, desde el punto de vista de la relación jurídico tributaria, tanto sustancial como formal, presenta serios desa-

\footnotetext{
* DOI: https://doi.org/10.18601/16926722.n11.06

${ }^{1}$ Contador Público y Especialista en Gerencia Tributaria, de la Universidad Surcolombiana, Magíster en Derecho con énfasis en Tributación, de la Universidad Externado de Colombia. Socio de Valbuena \& Lee Asociados. Correo-e: yhonyalee@ gmail.com

${ }^{2}$ Proyecto de Ley 053 del 03 de agosto de 2016. Cámara de Representantes.

${ }^{3}$ Proyecto de Ley 045 del 02 de agosto 2016. Cámara de Representantes.
} 
fíos conceptuales y prácticos que requieren ajustarse ya sea en futuras reformas tributarias, mediante decretos reglamentarios o vía doctrina oficial.

Palabras clave: Impuesto nacional al consumo, Impuesto Nacional al Consumo de Bolsas Plásticas - INCBP, Responsables, Sujeto pasivo económico, Sujeto pasivo jurídico, Monotributo.

\section{Abstract}

Law 1819 of 2016 in articles 207 and 208 regulated in its entirety the national tax on the consumption of plastic bags (hereinafter INCBP). The proposal of this new tribute, was not invention of the national government, since it was not considered in the initially presented project, but had like original creators to the Partido Alianza Verde, and specifically the senator Antonio Navarro Wolf, and to the Centro Democratico, who conceived it as a rate for the promotion of the environment, so that it was eventually included in the paper of the first debate and thus avoid a skill that could eventually become an unconstitutionality of this tax (Lee Yara, 2017).

The INCBP would appear to be a superfluous tax, since much of the discussion is considered unproductive, since taxpayers reduce it to a $\$ 20$ discussion. However, as we will demonstrate throughout this paper, from the point of view of the tax relationship, both substantial and formal process, presents serious conceptual and practical challenges that need to be adjusted either in future tax reforms, by regulatory decrees or via official doctrine.

Keywords: National Consumption Tax, National Consumption Tax Plastic Bags INCBP, Responsible, Economic Taxpayer, Legal Taxpayer, Monotributo.

\section{Tabla de abreviaturas}

DIAN Dirección de Impuesto y Aduanas Nacionales.

INCBP Impuesto Nacional al Consumo de Bolsas Plásticas.

INC Impuesto Nacional al Consumo.

IVA Impuesto al Valor Agregado.

\section{Introducción}

El Impuesto Nacional al Consumo de Bolsas Plásticas - INCBP es un impuesto pigouviano que tiene como finalidad corregir externalidades para contribuir a la mejora del medioambiente, motivando vía impuestos a que se reduzca el consumo de bolsas plásticas en el país, siendo este su principal objetivo.

Ahora bien, el hecho de que la tarifa del impuesto inicialmente sea de $\$ 20$ por bolsa, hace que desde la óptica de los sujetos pasivos económicos sea algo superfluo, considerando que no valga la pena un análisis 
jurídico del mismo por el impacto individual que este impuesto pueda tener.

No obstante, la relación jurídico tributaria, tanto sustancial como procedimental, como estructura de análisis de los impuestos, hace que una vez aplicada en el caso del INCBP surjan serios vacíos conceptuales o dudas al respecto.

Este estudio pretende analizar el hecho generador, los sujetos pasivos del impuesto, tanto económicos como jurídicos, la tarifa, la base gravable, el elemento espacial bajo las definición técnica de lo que debe entenderse como bolsa plástica, así como aspectos procedimentales, como la actualización del RUT, la obligatoriedad de presentar la declaración en ceros, su relación con el monotributo, su relación con el Impuesto sobre la Renta, entre otros.

La confrontación de la estructura con el INCBP dará como resultado un análisis crítico del impuesto nacional al consumo de bolsas prácticas - INCBP, que permite vislumbrar en el medio plazo un ajuste a la normatividad mediante la próxima reforma tributaria y, en el corto plazo, una aclaración ya sea vía decretos reglamentarios o doctrina oficial.

\section{Hecho generador}

El artículo 512-15 del Estatuto Tributario determina que el hecho generador del INCBP es la entrega a cualquier título de bolsas plásticas cuya finalidad sea llevar o cargar productos enajenados por los establecimientos comerciales. Con la delimitación del hecho generador del impuesto se pueden concluir varios aspectos relativos a este elemento de la relación jurídico-tributaria:
1. Que se trata de un hecho generador potencial, toda vez que lo que está gravando es un fin en sí mismo, como lo es llevar o cargar los productos enajenados. Con lo cual, en caso de que el sujeto pasivo económico manifieste al sujeto pasivo jurídico la negativa de la finalidad, estaríamos frente a un hecho económico sin relevancia en el mundo tributario, circunstancia descrita en el numeral 1 del artículo 512-16 del Estatuto Tributario como una no sujeción.

Al respecto, Hoyos y López (2017) describen con claridad esta problemática cuando señalan:

De esta exclusión podría entenderse que la finalidad con la que se adquiere la bolsa resulta ser el elemento determinante de la no sujeción, lo cual posiblemente genere dificultades, para su determinación, pues claramente el usuario podrá adquirir la bolsa y retirarla del establecimiento de comercio sin intención alguna hasta ese momento de utilizarla para cargar o llevar los productos adquiridos, reservando la materialización de la intención para otro momento, que no sea perceptible por la persona llamada a verificarla, lo cual tampoco está definido en la norma.

2. El segundo aspecto relativo al hecho generador es la entrega a título gratuito u oneroso. Pues en todo caso, independientemente de la forma como sea entregada la bolsa plástica, estamos frente a una "venta" para efectos del Impuesto al Valor Agregado - IVA, lo que implicaría que debería cobrarse igualmente este otro impuesto al gasto. Excepto, cuando la bolsa sea tratada como un activo fijo, pues este supuesto queda bajo el amparo del parágrafo 1 del artículo 420 del 
Estatuto Tributario ${ }^{4}$, como una venta de un activo fijo excluida de IVA.

Sobre este punto, la DIAN en su ABC del INCBP (DIAN) avaló la excepción cuando se trate de activos fijos. Situación que se presentaría de forma excepcional, toda vez que el artículo 21-1 del Estatuto Tributario incorporó los criterios de reconocimiento y medición de los nuevos marcos técnicos normativos de la contabilidad. Y en ellos, para que algo pueda ser considerado activo fijo antes de ser catalogado como un elemento, un activo debe cumplir con el principio de materialidad, que regularmente se da como un porcentaje del total de los activos fijos. Así las cosas, una vez observado el marco conceptual de la NIIF en lo ateniente al principio de materialidad (párrafo 2.6 de las NIIF para PYME y párrafo 7 de la NIC 1) difícilmente una bolsa podría llegar a ser considerada como un activo fijo. Con lo cual, podemos concluir que en la mayoría de los casos deberán cobrarse de forma concomitante tanto el INCBP como el IVA a la tarifa general del $19 \%$.

3. El tercer aspecto relativo al hecho generador del INCBP tiene que ver con la obligatoriedad de que sea un producto lo que sea adquirido. El inciso primero del artículo 512-15 del Estatuto Tributario, cuando hace referencia al hecho generador, indica que la finalidad de la bolsa sea cargar o llevar "productos enajenados por los establecimientos de comercio". Con lo cual, los servicios considerados en su integralidad, no generarían el INCBP para ningún responsable del INCBP.
4. El cuarto punto tiene que ver con la necesidad de contar con un establecimiento de comercio como un elemento determinante para la realización del hecho generador. Así las cosas, aun cuando se entreguen productos enajenados, si no se cuenta con un establecimiento de comercio no se realiza el hecho generador.

5. El artículo 512-16 del Estatuto Tributario contempla los casos de no sujeción. Dentro de ellos se resalta el numeral 2 relativo al preempaque de los productos, toda vez que los demás supuestos de no sujeción están condicionados a la reglamentación que expida el Gobierno Nacional. Al respecto, una parte de la doctrina ha señalado que "tratándose de bolsas de pre empaque, por el solo hecho de realizar el hecho generador quedan sujetas al INCBP, como es el caso de las bolsas transparentes usadas normalmente para empacar frutas y verduras" (Araque Cárdenas, Araque Durán, Varón García y Dussán Salazar, 2017, p. 327). Sin embargo, debe tenerse presente que aun cuando se realice el hecho generador, deben considerarse los supuestos de no sujeción, que para el caso específico está descrito en el numeral 2 del artículo 512-16 del Estatuto Tributario, y que conllevan a que la entrega de este tipo de bolsas, no tengan efectos tributarios.

6. Un aspecto final, que es quizá el más importante, tiene que ver con la diferenciación del INCBP como un impuesto autónomo e independiente, o si simplemente se trata de un nuevo hecho generador del Impuesto

${ }^{4}$ Dirección de Impuestos y Aduanas Nacionales, Oficio No 020901 del 4 de agosto 2017. 
Nacional al Consumo. Al respecto, algunos doctrinantes han considerado que se trata simplemente de una conservación del Impuesto Nacional al Consumo, pero que "agregó dos nuevos hechos generadores del tributo (i) la entrega a cualquier título de bolsas plásticas cuya finalidad sea cargar o llevar productos enajenados por los establecimientos comerciales que las entreguen y (ii) las ventas de productos transformados a partir de cannabis psicoactivo o no psicoactivo" (Plazas Vega, 2017) (destacado fuera del original). Es decir, se considera simplemente un nuevo hecho generador del INC.

Esta primera tesis tiene interrogantes que difícilmente resisten un debate académico, como, por ejemplo, si es simplemente un nuevo hecho generador y no un nuevo impuesto. ¿Por qué se incluyeron en dos artículos independientes y no dentro del artículo 512-1 que regula los hechos generadores del impuesto nacional al consumo? ¿Por qué tiene una denominación propia como INCBP? ¿Por qué redefinir los sujetos pasivos, tarifa, hecho generador y base gravable de forma independiente en un articulado propio?

Por otro lado, está la tesis de que se trata de un impuesto autónomo, que si bien tiene referencias a otros impuestos, como el IVA cuando señala los responsables, o el Impuesto Nacional al Consumo cuando señala el hecho generador, no se desvirtúa su autonomía e independencia. Esta postura está avalada vía doctrina mediante Oficio DIAN No 017806 del 06 de julio de 2017, donde se indica:

Tanto al impuesto de consumo de bolsas plásticas como el de cannabis, no existe ninguna remisión en la Ley 1819 de 2016 en sus artículos 207 a 213 que regularon estos dos impuestos, al régimen simplificado del impuesto nacional al consumo, por lo cual no resulta aplicable a estos impuestos.

Lo anterior evidencia la independencia con la cual cuenta este impuesto, pues señala que al no existir una remisión expresa no pueden aplicarse disposiciones de otros impuestos.

Esta segunda tesis tiene un solo interrogante que deja en serias dificultades el argumento de la independencia: ¿si el INCBP es independiente del impuesto nacional al consumo, por qué motivo se declaran en un mismo formulario como lo es el 310? Definir este último punto tiene efectos directos sobre el sujeto pasivo y sobre las obligaciones formales. Temas que analizaremos adelante.

\section{Sujeto pasivo económico}

De conformidad con el artículo 512-15 del Estatuto Tributario, el sujeto pasivo económico es quien soporta la carga financiera del impuesto como resultado de la realización del hecho generador, esto es, quien destina parte de su patrimonio para el pago del impuesto por la realización de los presupuestos descritos por la norma, que en el caso del INCBP es la persona que opte por recibir la bolsa. Lo que en principio no presenta dificultades interpretativas.

Sin embargo, en la parte final de la calificación del sujeto pasivo económico se señala que incluye domicilios. Así las cosas, consideramos que el domicilio debió ser incluido en la descripción del hecho generador, y no en la descripción del sujeto pasivo económico. Ya que, básicamente, 
no se tendría la posibilidad de decidir, pues una vez solicitado el domicilio y obviado el tema de las bolsas, la única manera de que no se genere este impuesto sería que se desempaque el domicilio y devuelva la bolsa al mensajero.

Sobre este aspecto, es preciso aclarar que aun cuando las ventas sean efectuadas mediante catálogos y/o internet, ambos conceptos quedan circunscritos a la definición de domicilios. Y en esa medida si se dan los demás elementos del impuesto, nada impide que se deba cobrar, excepto por el numeral 2 del artículo 512-16 del Estatuto Tributario relativo a la no sujeción del INCBP por preempaques.

\section{Sujeto pasivo jurídico}

La norma señala que son responsables de este impuesto las personas naturales o jurídicas que pertenezcan al régimen común de IVA. Lo que hace que bajo una primera lectura, no se observen aspectos críticos a comentar.

Ahora bien, en el IVA y en el Impuesto Nacional al Consumo aplican dos tipos de responsables: los del régimen simplificado y los del régimen común, cada uno de los cuales debe verificar los requisitos dependiendo del impuesto.

En el caso del INCBP, la norma no indica que hayan de aplicarse dichos regímenes. Con lo cual en un primer momento todas las personas naturales o jurídicas que pertenezcan al régimen común del IVA son responsables del INCBP, ergo todos pertenecerían a un solo régimen, que se consideraría un régimen general.
No obstante, tal como lo evidenciamos previamente en los comentarios efectuados al hecho generador, tomar el INCBP como un impuesto autónomo e independiente o como un hecho generador agregado al previamente constituido Impuesto Nacional al Consumo, hace que se presenten diferencias en relación con el sujeto pasivo jurídico.

Se reitera que si el INCBP se considera como autónomo e independiente, no deberían aplicarse los regímenes común y simplificado que operan en el Impuesto Nacional al Consumo, sino simplemente un régimen de responsabilidad general. Mientras que si se considera el INCBP como un nuevo hecho generador del Impuesto Nacional al Consumo, sí son compatibles, los dos regímenes (común y simplificado) señalados en los artículos y 512-14 del Estatuto Tributario, bajo el principio universal del Derecho de indica que el que puede lo más, puede lo menos. Pues si el INC regula todos los aspectos del impuesto, con mayor razón podrá regular los responsables y calificarlos, de uno de sus nuevos hechos generadores.

La DIAN ya tomó partido por la tesis de la independencia y autonomía, mediante Concepto 005311 de 2017, donde señaló lo siguiente:

3. ¿Para el Impuesto Nacional al Consumo de Bolsas Plásticas existe el régimen simplificado?

Sobre el particular, le precisamos que el inciso 5 o del artículo 207 de la Ley 1819 de 2016 incorporado con el artículo 512-15 del Estatuto Tributario señala de manera clara y sucinta que "son responsables de este impuesto las personas naturales y jurí- 
dicas que pertenecen al régimen común de IVA. (Lee Yara, www.gerencie.com, 2017).

En un primer momento, la doctrina parece no ser suficiente para superar este tema. No obstante, posteriormente la DIAN, mediante Oficio $\mathrm{N}^{\mathrm{o}} 017806$ del 06 de julio de 2017, dispuso:

Tanto al impuesto de consumo de bolsas plásticas como el de cannabis, no existe ninguna remisión en la Ley 1819 de 2016 en sus artículos 207 a 213 que regularon estos dos impuestos, al régimen simplificado del impuesto nacional al consumo, por lo cual no resulta aplicable a estos impuestos.

Así las cosas, bajo la tesis de impuesto autónomo, se evidencia que no existe el régimen simplificado del INCBP. Pero reiterando los argumentos esbozados en el análisis del hecho generador, valdría la pena preguntarse si es un impuesto "autónomo e independiente", ¿por qué se declara en el formulario 310 reservado para el Impuesto Nacional al Consumo - INC?

En uno y otro caso, sea del régimen común o del régimen simplificado, en caso de que este último se adopte, no aplica el INCBP en cuando a pesar de entregarse bolsas al sujeto pasivo económico lo haga en desarrollo de un contrato de prestación de servicios. Y en esa medida al no realizarse el hecho generador, no tiene consecuencias tributarias la entrega de dicha bolsa.

\section{La base gravable}

Sobre este punto, la doctrina nacional (Insignares Gómez, 2015) señala que "pueden existir impuestos de cuota fija o cuya base gravable no está determinada en términos monetarios sino en aspectos cuantitativos diferentes (por ejemplo, un contrato, una operación, entre otros)". Para este caso, es la bolsa en su individualidad.

Así las cosas, la base gravable es la cantidad de bolsas entregadas en cumplimiento del hecho generador.

\section{La tarifa}

Para el año 2017, "la tarifa es de \$20, para el año 2018 de \$30, para el año 2019 de $\$ 40$, para el año 2020 de $\$ 50$, y las mismas se actualizarán anualmente, en un porcentaje equivalente a la variación del índice de precios al consumidor certificado por el DANE al 30 de noviembre y el resultado se aproximará al peso más cercano”. Lo anterior igualmente estará avalado por certificación de la DIAN.

A su turno, el artículo 512-15 del Estatuto Tributario contempla unas tarifas diferenciales del $0 \%, 25 \%, 50 \%$ y $75 \%$, sobre el valor de la tarifa general, dependiendo del nivel de impacto. Pero está sujeta a reglamentación, lo cual no se ha dado a la fecha en que se escribe este artículo.

\section{El elemento espacial}

La norma no identifica con claridad el elemento espacial del INCBP. Tal como se indicó en lo relativo al hecho generador, la finalidad se ubica al momento de causación, esto es, la entrega de la bolsa.

Ahora bien, el elemento espacial del impuesto adquiere vital importancia bajo una definición técnica de lo que debe entenderse 
como bolsa plástica. Sobre este punto, es menester aclarar que la Resolución 0668 del 28 de abril de 2016 emitida por el Ministerio del Medio Ambiente y Desarrollo Sostenible, define como bolsa plástica aquella "distribuida en los puntos de pago". Con lo cual, si una bolsa plástica se entrega en un sitio diferente al punto de pago, no se realizaría el hecho generador del impuesto y puntualmente por el elemento especial definido por una norma técnica especial.

Este hecho no tendría discusión alguna si se hubiese dado claridad sobre el elemento especial desde el punto de vista tributario, pero que en ausencia de ello, al recurrir a una definición técnica, haría que cualquier bolsa entregada en un punto distinto al punto de pago pueda entenderse como un hecho económico, sin relevancia tributaria, por no realizarse el hecho generador.

\section{Otras consideraciones}

\section{Contabilización y su relación con el Impuesto sobre la Renta}

Este impuesto no podrá tratarse como costo, deducción o impuesto descontable, de conformidad con el inciso sexto del artículo 512-15 del Estatuto Tributario. Con lo anterior se generaría un gasto de naturaleza contable que tiene la característica de no ser tratado como costo o deducción en el Impuesto sobre la Renta, y que en consecuencia se convierte en un impuesto no recuperable.

Lo anterior hace que el adquiriente, en caso de que la bolsa haga parte de un producto que se pretende reconocer como activo en un momento inicial, pueda ser reconocido como parte del costo de adquisición del ac- tivo (bajo el modelo del costo en NIIF para PYMES), al ser un impuesto no recuperable.

\section{La factura o documento equivalente}

La norma indica que, en todos los casos, en la factura de compra o documento equivalente deberá constar expresamente el número de bolsas y el valor del impuesto causado. Así las cosas, tanto el número de bolsas, como el impuesto causado deben ser parte integral de la factura de forma expresa. No pudiendo confundirse con IVA o con otros elementos como la base del impuesto. En este sentido, es recomendable que los departamentos de facturación y sistemas tomen las decisiones pertinentes, so pena de incurrir en una conducta sancionable por expedir facturas sin el cumplimiento de los requisitos legales.

\section{Monotributo}

El parágrafo 3 del artículo 512-16 del Estatuto Tributario señala que los sujetos pasivos del monotributo podrán acogerse voluntariamente al INCBP. En este caso, el monto del INCBP se entenderá incluido en la tarifa fija del monotributo.

A pesar de que la norma no indica la calidad en que se pueda acoger, si como sujetos pasivos económicos o sujetos pasivos jurídicos. Debe entenderse que se acogerían como sujetos pasivos jurídicos. Toda vez que indica que estará incluido en la tarifa del monotributo, y solo los sujetos pasivos jurídicos son los que transfieren lo recaudado al fisco.

Este aspecto presenta serios desafíos conceptuales, pues los sujetos pasivos del monotributo deben cumplir las siguientes 
características, de conformidad con el artículo 905 del Estatuto Tributario:

- Ser personas naturales.

- Que en el año gravable hubieren obtenido ingresos brutos ordinarios o extraordinarios, iguales o superiores a 1.400 UVT e inferiores a 3.500 UVT.

- Que desarrollen su actividad económica en un establecimiento con un área inferior o igual a 50 metros cuadrados.

-Que sean elegibles para pertenecer al Servicio Social Complementario de Beneficios Económicos Periódicos - BEPS.

- Que tengan como actividad económica una o más de las incluidas en la división 47 comercio al por menor y la actividad 9602 peluquería y otros tratamientos de belleza de la Clasificación de Actividades Económicas - CIIU adoptada por la Dirección de Impuestos y Aduanas Nacionales - DIAN.

Una vez revisados estos requisitos, al contrastarlos con los que señala el artículo 499 del Estatuto Tributario, se observa que algunas personas naturales pueden pertenecer al régimen simplificado del IVA o inclusive no tener régimen aplicable en el IVA por no realizar actividades gravadas, pero sí llegar a ser calificadas como sujetos pasivos del monotributo. Y ya calificado como sujeto pasivo del monotributo, el parágrafo 3 del artículo 512-15 del Estatuto Tributario, lo habilita para ser sujeto pasivo del INCBP.

Es decir, personas naturales que no pertenecen al régimen común del IVA (como es la regla general consagrada en el inciso 5 del artículo 512-15 del Estatuto Tributario) puedan ser sujetos pasivos del INCBP y, en consecuencia, recaudar el valor del impuesto.

Lo anterior, aunado al hecho de que una vez cobrado este impuesto por parte del sujeto pasivo del monotributo, constituiría un enriquecimiento, ya que lo pagado por los sujetos pasivos económicos no sería transferido al Estado, sino que al estar incluido dentro de la tarifa del monotributo, enriquecería a un particular con un impuesto del orden nacional. Si bien no es representativo en términos monetarios, sí lo es jurídicamente y más exactamente en el plano conceptual, pues desnaturaliza en principio la tipología tributaria de impuesto, y en segundo grado la destinación de los impuestos. A partir de lo anterior, este apartado puede considerarse abiertamente inconstitucional.

\section{Declaración}

Partiendo de la teoría de la independencia tendríamos que son sujetos pasivos las personas naturales y jurídicas responsables del régimen común del IVA. Pero, ¿están en la obligación de declarar todos los responsables del INCBP?

Pues bien, una primera tesis indica que no se debe presentar la declaración, toda vez que al no realizar el hecho generador (entiéndase la entrega de la bolsa plástica con la finalidad indicada) no se está en la obligación de presentar la declaración. Aunado a lo anterior, el Consejo de Estado indicó, en el caso del impuesto de renta y complementarios, que "para estar obligado a declarar, se debe previamente realizar el hecho generador del impuesto" (Sentencia 16028 de 2008). En esa medida, al no entregarse 
bolsas con la finalidad dispuesta, no se está en la obligación de presentar la declaración.

Contrario a ello, está la tesis de la responsabilidad, que indica que con el calificativo de responsables del INCBP, y sin norma alguna que subrogue al responsable de presentar la declaración en el periodo que no se efectuaron operaciones sujetas al impuesto, debe presentarse la declaración aun cuando sea en ceros. Esta tesis tiene como fundamentos los antecedentes del IVA que tan solo con norma expresa contenida en la Ley 1430 de 2010, eliminó la obligación de presentar la declaración en ceros. Adicionalmente, en el caso del Impuesto de Renta, el Estatuto Tributario contempla los artículos 591 y ss., que determinan parámetros para estar obligado a presentar la declaración con indicadores patrimoniales y de consumo, sin que se tenga en cuenta la realización del hecho generador. Es decir, aun cuando no se perciban ingresos (hecho generador) se puede estar obligado a declarar (por patrimonio o consumo de tarjetas, consignaciones bancarias, entre otros.). Se reitera que solo mediante una norma expresa se elimina la obligación de presentar la declaración correspondiente.

Así las cosas, la DIAN, en relación con el Impuesto Nacional al Consumo - INC, señaló en el Oficio 17357 del 4 de julio de 2017 que cuando no se realicen operaciones sujetas al impuesto no se está en la obligación de presentar la declaración. Debe aclararse que este pronunciamiento solo hace referencia al Impuesto Nacional al Consumo - INC, y no hace mención al Impuesto Nacional al Consumo de Bolsas Plásticas.

Ahora bien, considerando que mediante el acto administrativo que adopta los formula- rios aplicables al año 2017 (Resolución DIAN 000013 del 03 de marzo de 2017), y específicamente el anexo de formulario, se indica que el Impuesto Nacional al Consumo de Bolsas Plásticas - INCBP debe declararse en el formulario 310, originalmente consagrado para el Impuesto Nacional al Consumo - INC, debe entenderse que en todo lo referente a la declaración del INC debe aplicarse igualmente al INCBP. En consecuencia, al ser el formulario 310 el aplicable igualmente al INC e INCBP, si no se realiza el hecho generador, no se está en la obligación de presentar la declaración en ceros.

Obsérvese como la DIAN, si bien previamente parecía tener claro que el INCBP es un impuesto independiente del INC, para efectos de la presentación de la declaración une los dos impuestos en el formulario 310. Lo cual puede entenderse como una medida de simplicidad formal. Pero que también podría entenderse como un supuesto integrador, pues al solo incluirse en unos renglones específicos y no en una declaración propia, puede concluirse que el INCBP es un hecho generador adicional del INC y no un impuesto autónomo e independiente.

En el caso de que eventualmente admitiéramos que puede llegar a existir el régimen simplificado del INCBP (ver explicación del sujeto pasivo jurídico) bajo una teoría integradora que determine este impuesto como un simple hecho generador adicional y no como un impuesto verdaderamente autónomo e independiente, en lo concerniente a la declaración, la Ley 1819 de 2016 adicionó al Estatuto Tributario el artículo 512-14 Estatuto Tributario, que impuso al régimen simplificado del INC, las obligaciones contenidas en el artículo 506 del Estatuto Tributario, es 
decir, las relativas al régimen simplificado del IVA. Nótese que este artículo solo habla del Impuesto Nacional al consumo general y no del INCBP.

Basado en ello, la DIAN, en Oficio 017806 del 6 de julio de 2017, señaló que a partir del 1 de enero de 2017 el régimen simplificado del INC no debe presentar la declaración del formulario 315 , contrario a lo que había lo dispuesto en la Resolución 000006 del 3 de febrero de 2017, donde señaló que "todos" los responsables del régimen simplificado del INC deben presentar la declaración.

\section{Actualización del RUT}

La correlativa calificación de la responsabilidad del INCBP implica necesariamente la actualización del RUT para incluir la responsabilidad 33 Impuesto Nacional al Consumo, so pena de incurrir en una sanción por no actualización del RUT.

\section{El cambio comercial}

Las operaciones comerciales representan en todo sentido la materialización de los hechos económicos que sirven de base para calificar los hechos generadores del impuesto. De allí que la práctica mercantil determine en gran medida el éxito de las medidas tributarias.

En ese sentido, el gran problema a nivel comercial ha sido la no disposición de monedas de baja denominación para proceder a efectuar el cambio a los sujetos pasivos económicos. Lo anterior, ha hecho que las empresas terminen asumiendo este impuesto o en su defecto que se cobre por un monto superior, generando ganancias que, acu- muladas, representan un mayor valor vía impuesto de renta.

Con todo, al menos en los cálculos del Banco de la República existen suficiente monedas de baja denominación en el mercado. Y en todo caso, tal como lo señala la DIAN en oficio No 0209004 de agosto 2017, ello no constituye un impedimento para el recaudo del IINCBP.

\section{Régimen sancionatorio}

Los artículos 512-15 y 512-16 del Estatuto Tributario nada dicen respecto del régimen sancionatorio aplicable. No obstante, toda vez que el INCBP se presenta en el formulario 310 relativo al INC, consideramos que el régimen sancionatorio aplicable es el del INC, sin que ello implique una afectación al principio de legalidad en materia sancionatoria.

\section{Conclusiones}

Tal como se pudo evidenciar a lo largo de este escrito, si bien para algunos las discusiones del INCBP pueden reducirse a $\$ 20$, al menos desde el plano académico y práctico se presentan serias dificultades conceptuales que hacen que sea necesaria una revisión a partir de la teoría de la relación jurídicotributaria, tanto en los aspectos sustanciales como en los procedimentales. De lo contrario, tendremos conceptos jurídicos indeterminados que atentan contra el principio de seguridad jurídica que debe irradiar nuestro sistema tributario.

Desde el punto de vista del control tributario, la reforma es ajena a ello, toda vez que la DIAN no podrá identificar la cantidad de bolsas entregadas, y si ellas cumplen con la 
finalidad contenida en el hecho generador, lo que hace que los procedimientos de fiscalización en el INCBP sean nulos. La medida solo podrá ser eficaz si los sistemas de la DIAN logran enlazarse con los datos que reportan una parte de los sujetos pasivos jurídicos al Ministerio del Medio Ambiente y Desarrollo Sostenible, pues es dicha entidad en la que reposan datos respecto del consumo de bolsas plásticas en el país.

Por otro lado, la finalidad del INCBP consistente a la reducción del consumo de bolsas plásticas, se ha cumplido a cabalidad, toda vez que durante el primer trimestre del año 2017 se ha evidenciado una reducción en el consumo de dichas bolsas de un 25\% (Dinero, 2017).

Finalmente, el hecho de que se estén logrando las metas en disminución del consumo de bolsas plásticas, no debe considerarse como una excusa para un análisis más riguroso del INCBP desde el punto de vista técnico y jurídico, que permita que esta herramienta corrija las externalidades negativas dentro de un marco tributario legítimo. 


\section{Bibliografía}

Araque Cárdenas, J. H.; Araque Durán, J. D.; Varón García, L. y Dussán Salazar, W. (2017). Nueva reforma tributaria ley 1819 de 2016 explicada. Bogotá: Nueva Legislación.

DIAN (s.f.). www.dian.gov.co. Recuperado el 17 de Octubre de 2017, de DIAN: http://www.dian.gov.co/descargas/ centrales/2017/Abece_Impuesto_bolsas.pdf

Dinero (03 de 08 de 2017). Recuperado el 31 de 10 de 2017, de www.dinero.com: http://www.dinero.com/ edicion-impresa/pais/articulo/bolsa-plastica-baja-el-consumo-de-bolsas-plasticas/248180

Hoyos, V. y López, M. (2017). Impuesto Nacional al Consumo en el marco de la Reforma Tributaria Estructural Ley 1819 de 2016. En J. R. Piza Rodríguez, Análisis Critico de la Reforma Tributaria Estructural Ley 1819 de 2016 (págs. 485-494). Bogotá: Universidad Externado de Colombia .

Insignares Gómez, R. (2015). Principios constitucionales del sistema tributario colombiano. En J. R. Piza , La obligación tributaria y sus fundamentos constitucionales. Bogotá: Editorial Universidad Externado de Colombia.

Lee Yara, Y.A. (15 de septiembre de 2017). www.gerencie.com. Recuperado el 17 de octubre de 2017, de Gerencie. com: https://www.gerencie.com/hubo-habilidosidad-en-el-impuesto-nacional-al-consumo-de-bolsas-plasticas.html

Lee Yara, Y. A. (03 de agosto de 2017). www.gerencie.com. Recuperado el 17 de 10 de 2017, de Gerencie.com: https://www.gerencie.com/existe-el-regimen-simplificado-en-el-impuesto-nacional-al-consumo-de-bolsasplasticas.html

Plazas Vega, M.A. (2017). La Ley 1819 de 2016 en lo que concierne al impuesto sobre el valor agragado (IVA) y al impuesto nacional al concumo (INC). En J. G. Ruiz Hurtado, Comentarios a la reforma tributaria estructural ley 1819 de 2016 (pág. 248). Bogotá: Instituto Colombiano de Derecho Tributario .

\section{Jurisprudencia}

Consejo de Estado, Sección Cuarta (17 de abril de 2008) Sentencia 16028. M.P.: Ligia López Díaz.

\section{Leyes y Proyectos de Ley}

Congreso de Colombia (29 de diciembre de 2016). Por medio de la cual se adopta una reforma tributaria estructural, se fortalecen los mecanismos para la lucha contra la evasión y la elusión fiscal, y se dictan otras disposiciones. Ley 1819 de 2016, DO: 50.101, artículos 207 y 208.

Cámara de Representantes (02 de agosto 2016). Proyecto de Ley 045 de 2016.

Cámara de Representante (03 de agosto de 2016). Proyecto de Ley 053 de 2016.

Estatuto Tributario, parágrafo 1 del artículo 420.

\section{Resoluciones}

Dirección de Impuestos y Aduanas Nacionales (03 de febrero de 2017). Resolución 000006 del 03 de febrero de 2017.

Dirección de Impuestos y Aduanas Nacionales (03 de marzo de 2017). Resolución 000013 del 03 de marzo de 2017.

Ministerio del Medio Ambiente y Desarrollo Sostenible. (28 de abril de 2016) Resolución 0668 del 28 de abril de 2016. 


\section{Doctrina}

Dirección de Impuestos y Aduanas Nacionales. Oficio 015172 de 2017.

Dirección de Impuestos y Aduanas Nacionales. Oficio 020897 de 2017.

Dirección de Impuestos y Aduanas Nacionales. Oficio 020901 de 2017.

Dirección de Impuestos y Aduanas Nacionales. Oficio 020900 de 2017.

Dirección de Impuestos y Aduanas Nacionales. Oficio 017906 de 2017.

Dirección de Impuestos y Aduanas Nacionales. Oficio 017806 de 2017.

Dirección de Impuestos y Aduanas Nacionales. Oficio 017357 de 2017.

Dirección de Impuestos y Aduanas Nacionales. Oficio 016243 de 2017.

Dirección de Impuestos y Aduanas Nacionales. Oficio 005963 de 2017.

Dirección de Impuestos y Aduanas Nacionales. Oficio 005311 de 2017. 